\title{
Enhanced High-Rate Shockwave Lithotripsy Stone Comminution in an In Vivo Porcine Model Using Acoustic Bubble Coalescence
}

\author{
Hedieh Alavi Tamaddoni, MSE, BS, William W. Roberts, MD, Alexander P. Duryea, PhD, ${ }^{3}$ \\ Charles A. Cain, PhD, and Timothy L. Hall, $\mathrm{PhD}^{1}$
}

\begin{abstract}
Cavitation plays a significant role in the efficacy of stone comminution during shockwave lithotripsy (SWL). Although cavitation on the surface of urinary stones helps to improve fragmentation, cavitation bubbles along the propagation path may shield or block subsequent shockwaves (SWs) and potentially induce collateral tissue damage. Previous in vitro work has shown that applying low-amplitude acoustic waves after each SW can force bubbles to consolidate and enhance SWL efficacy. In this study, the feasibility of applying acoustic bubble coalescence $(\mathrm{ABC})$ in vivo was tested. Model stones were percutaneously implanted and treated with 2500 lithotripsy SWs at $120 \mathrm{SW} /$ minute with or without $\mathrm{ABC}$. Comparing the results of stone comminution, a significant improvement was observed in the stone fragmentation process when $\mathrm{ABC}$ was used. Without $\mathrm{ABC}$, only $25 \%$ of the mass of the stone was fragmented to particles $<2 \mathrm{~mm}$ in size. With $\mathrm{ABC}, 75 \%$ of the mass was fragmented to particles $<2 \mathrm{~mm}$ in size. These results suggest that $\mathrm{ABC}$ can reduce the shielding effect of residual bubble nuclei, resulting in a more efficient SWL treatment.
\end{abstract}

Keywords: acoustic bubble coalescence, extracorporeal shock wave lithotripsy, high rate SWL, in vivo, renal stone, shielding effect

\section{Introduction}

A STRONG RATE DEPENDENCE in the efficacy of stone comminution for shockwave lithotripsy (SWL) has been shown by many in vitro, ${ }^{1-4}$ in vivo,${ }^{5}$ and human studies. ${ }^{6-8}$ It is hypothesized that a major component of this effect is due to the presence of cavitation bubbles persisting from shockwave (SW) to SW that shields or attenuates the amplitude of subsequent lithotripter SWs. ${ }^{9-11}$ At low firing rates, there is sufficient time for a majority of the bubbles to passively dissolve, while at high firing rates, the per SW efficacy is significantly reduced due to persisting bubbles.

The fragmentation of renal calculi by SWL is understood to be produced through mechanical stresses caused by a combination of incident SWs and the collapse of cavitation bubbles on the surface of the urinary stones. ${ }^{12,13}$ Although mechanical stress generated by the incident SW causes the initial disintegration of the stone, cavitation is a necessary component for efficient fragmentation, and particularly for producing fine passable fragments $(<2 \mathrm{~mm}) .{ }^{13}$ The effect of cavitation on the fragmentation process is thought to be primarily through erosion on the stone surface, but it may also help in the formation of crack lines through which damage is spread from the surface into the bulk of the stone. This damage on the surface of the stone makes the structure weaker and as a result more susceptible to subsequent SWs. ${ }^{13-15}$

The growth and collapse lifespan of a cavitation bubble cloud produced by a lithotripter SW has been shown to be on the order of 1 millisecond, much shorter than the interval between SWs at typical lithotripter firing rates of $0.5-2 \mathrm{~Hz}$. However, the residual micron-sized bubbles following a cavitation cloud collapse have a longer lifespan on the order of 1 second and thus a large fraction would be expected to persist between subsequent SWs, particularly at higher firing rates. ${ }^{2,16-18}$ On subsequent pulses, these nuclei absorb energy from the negative pressure phase of the lithotripter waveform, reducing cavitation on the surface of the stone, which in turn reduces the efficacy of stone comminution. ${ }^{2}$

In our previous studies, low-amplitude acoustic bursts were used with in vitro models to actively remove residual cavitation bubbles through forced coalescence.,19,20 Significant improvement was demonstrated in the comminution efficacy of SWL at higher rates (120 and $60 \mathrm{SW} /$ minute). In this study, the feasibility of SWL stone comminution at 120 
$\mathrm{SW} /$ minute with acoustic bubble coalescence (ABC) was evaluated on a porcine model and compared to standard SWL at the same rate.

\section{Materials and Methods}

After IACUC approval, a total of twelve $45-50 \mathrm{~kg}$ female pigs were used in this study. Throughout the procedure, the pigs were anesthetized with $2 \%-2.5 \%$ inhaled isoflurane, while their vital signs, including body temperature, heart rate, oxygen saturation, and respiration, were monitored by a Vet/Ox Plus 4800 Vital Signs Monitor (Heska, Loveland, CO). Each pig was placed prone on a surgical table. Flexible cystoscopy (Olympus CYF-3) was performed to visualize and cannulate the right ureter with an access wire. An $8 \mathrm{~F}$ silicone urinary catheter was passed partially up the ureter and the balloon inflated with 5-10 cc saline to produce ureteral occlusion. One hour later, the animal was repositioned semiprone (left flank up) and transcutaneous ultrasound (Ultrasonics, Richmond, Canada) confirmed hydronephrosis. With ultrasound guidance, percutaneous access was obtained above the 12th rib to enter the collecting system through an upper pole renal calix. An angled glide wire was advanced into the collecting system and manipulated down the ureter, then replaced with an Amplatz stiff wire, over which sequentially dilation was performed up to 26F and a sheath placed. Through this sheath, endoscopy of the collecting system was performed using the flexible ureteroscope. Once the designated lower pole calix (occasionally a mid-renal calix was used) was identified, the cystoscope was removed and reloaded with a zero-tip $1.9 \mathrm{~F}$ wire basket grasping a $6 \mathrm{~mm}$ diameter cylindrical stone. The cystoscope and basket were passed through the sheath into the collecting system and navigated into the previously identified lower pole calix where the stone was released. Any air introduced during implantation was aspirated with the flexible cystoscope, while flushing degassed water through the ureteral access catheter.

The model stones were cylindrical in shape, $6 \mathrm{~mm}$ in diameter, and about $7 \mathrm{~mm}$ in height, with an average hydrated mass of $281.7 \mathrm{mg}(\mathrm{std}=33.8 \mathrm{mg})$. The stones were made by a mixture of BegoStone plaster (BEGO, Smithfield, RI), al- bumin (Carolina Biological Supply Co., Burlington, NC), and water using the method described by Simmons and colleagues $^{21}$ formed in an acetal mold. The mass ratio of mixture was $73 \%$ BegoStone, $2.5 \%$ albumin, and $24.5 \%$ water. Model stones were formulated to imitate the structural strength of cysteine composition stones. The stones were stored in degassed water under vacuum for at least 24 hours before use to minimize any gas incorporated during formation.

After the stone was implanted into the subject and air evacuated from the collecting system, the access sheath and wire were removed, and the incision was sealed with superglue. The occlusion catheter in the ureter was deflated to allow urinary drainage and the animal was left to rest for about 30 minutes before the treatment. The incision was not part of the subsequent acoustic window for the lithotripsy treatment. The skin over the targeted area was shaved and treated with depilatory cream to improve ultrasound coupling. The location of the target was marked on the skin and a water bolus consisting of a large plastic bag was placed on the pig over the targeted area. To ensure optimal sound coupling, the bolus was glued directly to the skin around a $15 \mathrm{~cm}$ cutout hole so that there was no membrane between fluid and skin (equivalent to an immersion bath). The bolus was filled with degassed water at room temperature of about $21^{\circ} \mathrm{C}$ and the dissolved gas concentration maintained below $10 \%$ saturation by continuous recirculation and degassing. A stereotactic positioning setup was used where a curvilinear imaging array probe (C4 on P6 scanner; GE Medical Systems, Waukesha, WI) was positioned with a three-axis motorized system to target the implanted stone. The imaging probe assembly was then removed and replaced with the lithotripter head assembly where the focus was aligned to the ultrasound image.

A laboratory electrohydraulic lithotripter (EHL) was used for all the treatments modeled on the design of Coleman et al. $^{22}$ to simulate the acoustic field generated by a Dornier HM3 (Dornier Medical Systems, Kennesaw, GA). One modification was that the system used a straight downward firing configuration to facilitate rapid electrode changes. ${ }^{22,23}$ In addition, water was drawn continuously through the head via small ports at the highest point on the reflector and
FIG. 1. Experiment setup: the model stone implanted in the right kidney of the porcine model is targeted and treated by a laboratory electrohydraulic lithotripter patterned after Dornier HM3 along with a separate annular transducer array generating the $\mathrm{ABC}$ pulses. The water bolus is filled with degassed water with gas concentration below $10 \%$ saturation and contains a $15 \mathrm{~cm}$ cutout for direct coupling of sound from the water to the skin. The animal's vital signs, including oxygen saturation, heart rate, respiration, and core body temperature, were monitored throughout the experiment. $\mathrm{ABC}$, acoustic bubble coalescence.

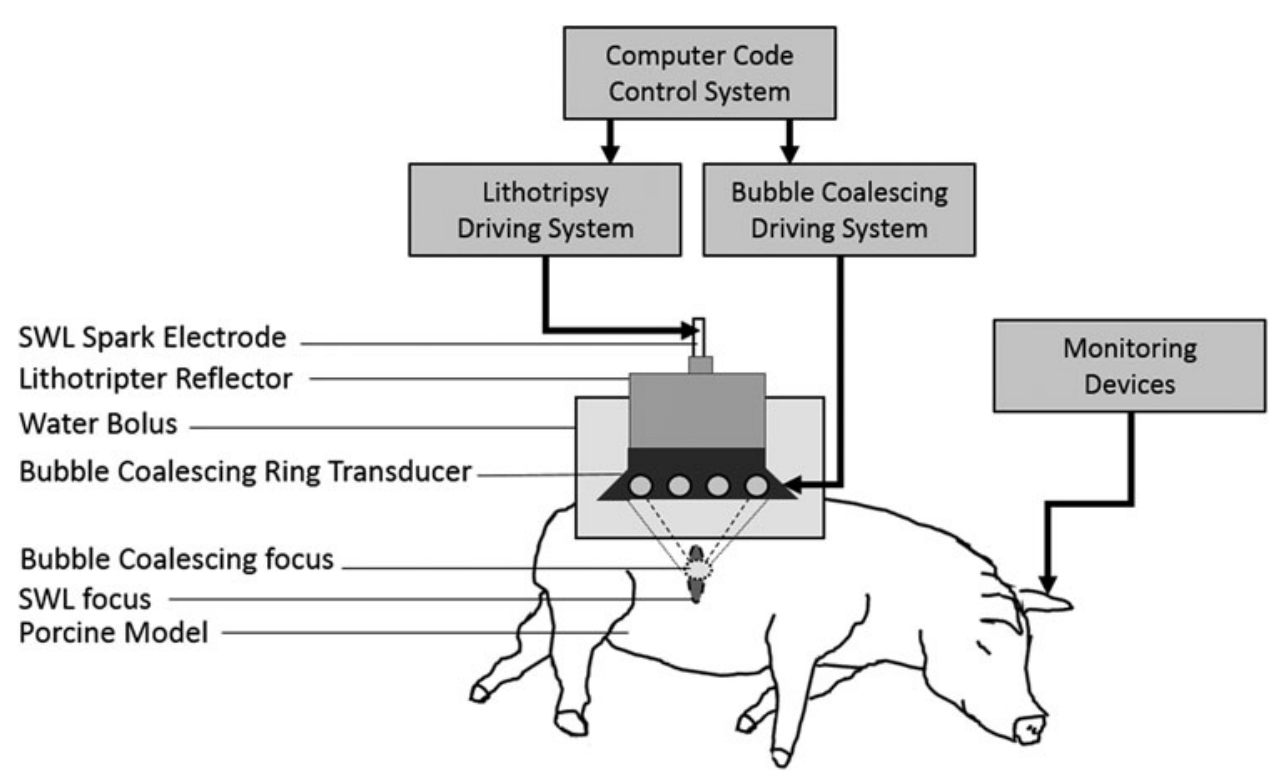




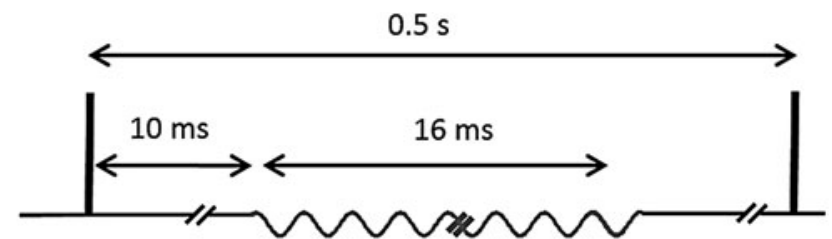

\section{Shockwave Bubble coalescence Pulses \\ $\mathrm{P}+/ \mathrm{P}-=34 \mathrm{MPa} / 8 \mathrm{MPa} \quad 1 \mathrm{MPa}, 500 \mathrm{kHz}$}

FIG. 2. Acoustic pulse sequence for SWL and bubble coalescing. The SWL rate was $120 \mathrm{SW} /$ minute interleaved with bubble coalescing pulses applied for duration of 16 millisecond. SW= shockwave; SWL= shockwave lithotripsy.

recirculated into the water bolus to remove any cavitation bubbles formed by the electrode. The lithotripter was operated at a firing rate of $120 \mathrm{SW} /$ minute and charging voltage of $20 \mathrm{kV}$, generating SWs with a peak positive pressure $(\mathrm{P}+)$ of $34 \mathrm{MPa}$ and a peak negative pressure $(\mathrm{P}-)$ of $8 \mathrm{MPa}$. All EHL electrodes (HealthTronics, Austin, TX) used in the experiments were preconditioned with 500 break-in shocks at $20 \mathrm{kV}$ and $120 \mathrm{SW} /$ minute the day before usage for maximum acoustic output. In each treatment, two electrodes were used to generate a total of 2500 shocks, 1250 uninterrupted shocks on each of the electrodes (Fig. 1).

$\mathrm{ABC}$ sequences were applied during five of the ten experiments interleaved between each SW. The ABC transducer was constructed in-house, consisting of an annular array of eight $500 \mathrm{kHz}$ transducers, each $50 \mathrm{~mm}$ diameter, surrounding the lithotripter reflector. ABC sequences consisted of alternating tone bursts of two cycles from each transducer at a pulse repetition rate of $100 \mathrm{kHz}$ and amplitude of $1 \mathrm{MPa}$ for a total duration of 16 millisecond. The bubble coalescence pulses were applied after a 10-millisecond delay to let the initial bubble cloud to grow and collapse first (Fig. 2) This pulse sequence was designed to provide a relatively uniform unfocused sound field from a ring transducer geometry similar to the one in our previous in vitro study. ${ }^{1}$

Posttreatment position of the targeted kidney stone was confirmed by removing the lithotripter head and reattaching the GE imaging probe assembly. The subject was then euthanized and the kidney harvested, including a portion of distal ureter. The kidney was carefully dissected and all stone fragments remaining in the collecting system were recovered by flushing with water and further dissection. Collected fragments were filtered through $4,2,1$, and $1 / 2 \mathrm{~mm}$ hole size perforated brass plates. The fragments of each bin size were dried for 48 hours and then weighed. Three control stones not
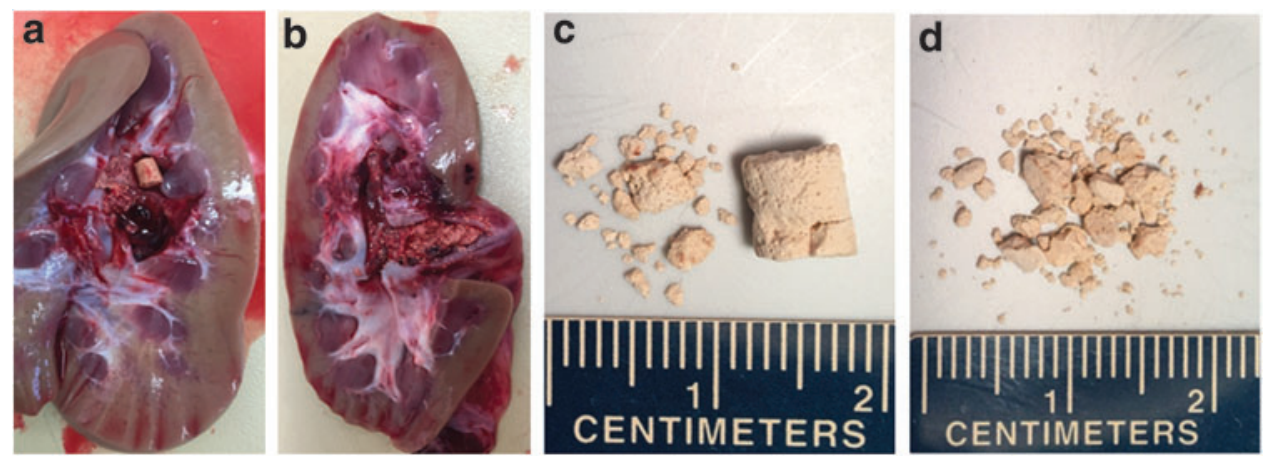

FIG. 3. Typical results from normal SWL treatment (a, c) and SWL with ABC (b, d). Recovered fragments from treatments with normal SWL (c) were visibly larger than treatments with SWL and $\mathrm{ABC}(\mathbf{d})$. 


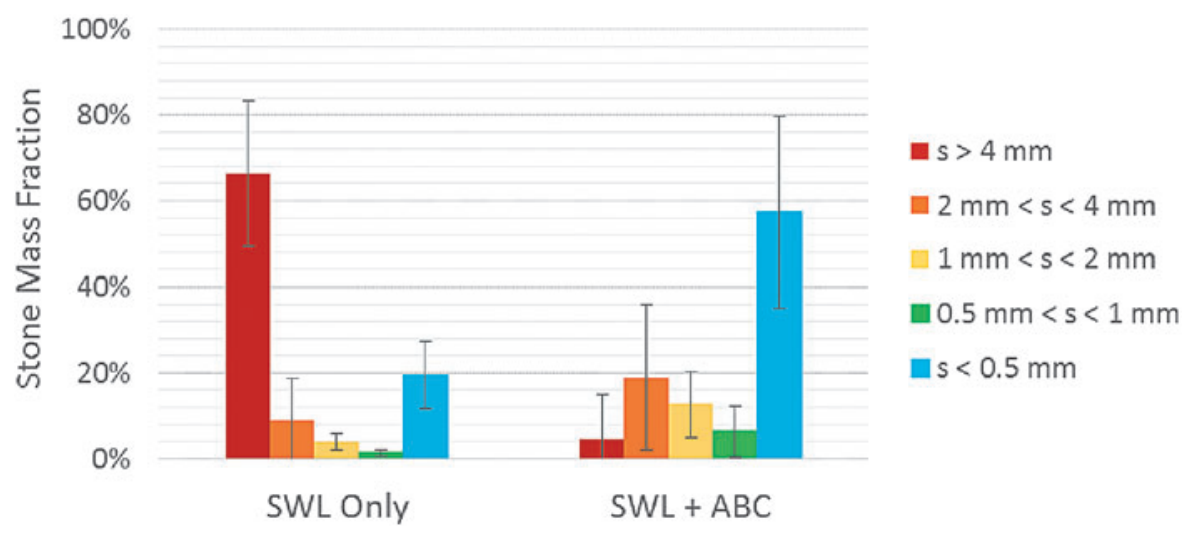

tendency of bubbles smaller (or larger) than resonant size to move up (down) a pressure gradient and congregate at pressure antinodes (or nodes). The secondary Bjerknes force describes an attractive force between bubbles oscillating in phase depending on their size and the acoustic field frequency. This secondary Bjerknes force is hypothesized to be the dominant mechanism bringing the remnant bubbles from SWL cavitation together to coalesce and clear the propagation path. ${ }^{1,20}$ In general, the degree of coalescence required for effective clearance of the propagation path to mitigate effects of bubble shielding is not known. This suggests the potential for further optimization of acoustic parameters for $\mathrm{ABC}$ for further improvement in fragmentation efficiency.

The SW rate used for this study was $120 \mathrm{SW} /$ minute, which is a common rate used in clinical SWL. This high rate was chosen to reduce experiment time and possibly enhance the difference between study groups. In vitro work ${ }^{1}$ has shown that a significant, although somewhat reduced, difference also occurs at $60 \mathrm{SW} /$ minute. The goal in this study was to understand the barriers to high-rate SWL and consider methods to alleviate them. Although, this study does not show that prefocal cavitation and the shielding effect is the only barrier to high-rate SWL, reducing the population of prefocal cavitation by means of forced coalescence appears to improve the efficiency of high-rate SWL significantly.

One of the major differences between distribution of stone fragments in subjects with and without $\mathrm{ABC}$ was the presence of at least one larger than $4 \mathrm{~mm}$ stone fragments in subjects without $\mathrm{ABC}$. In previous studies, we have shown that shielding effect reduces the cavitation on the surface of the stone, ${ }^{2}$ which is a significant factor in producing fine fragments by means of surface erosion and weakening the structure of stone that makes the further fragmentation to smaller pieces more probable during subsequent SWs. ${ }^{13-15}$ As a result, by removing prefocal cavitation from the propagation path and reducing the shielding effect, it was expected to see lower amount of large fragments when $\mathrm{ABC}$ was applied.

An additional problem with high-rate SWL is an increased risk for collateral tissue damage. Cavitation within the kidney parenchyma is thought to be the source of this damage. It is possible that $A B C$ with SWL could help reduce this damage by preventing a proliferation of cavitation bubbles expanding into the kidney tissue. However, it is also possible that cavitation damage could be exacerbated by additional forced oscillation of bubbles outside the collecting system. A sep- arate study of tissue damage in SWL with and without ABC will be required to assess the extent of tissue damage without the trauma associated with stone implantation.

Related work has shown improvement in stone comminution by interleaving SWL with auxiliary SWs produced by a piezoelectric annular array SW generator. ${ }^{24,25}$ The auxiliary SWs in the mentioned study are of extremely short duration and on the order of $8 \mathrm{MPa}$ pressure timed to arrive right after the initial collapse of bubble cloud produced by each lithotripter SW, generating a secondary intensified collapse near the surface of the stone. It is important to note the significant differences in the amplitude and duration of the acoustic fields used compared to this study. Interestingly, the acoustic sound sources have a remarkably similar geometry.

Optimal bubble coalescence requires a nonfocused acoustic field that exposes as much of the propagation path as possible, where cavitation may occur. Ultrasound-guided lithotripters with a central access port for imaging are ideally suited for ABC because the coalescence generator could be a simple small unfocused transducer. Systems lacking this port (generally X-ray guided) require a slightly more complex annular array transducer to generate the coalescence field such as the device demonstrated in this study, but is still possible without modification of the SW source.

\section{Conclusions}

This study demonstrates the feasibility of utilizing ABC in enhancing high-rate SWL. The results suggest significant improvement in the stone fragmentation process when using bubble coalescence with SWs, which implies that ABC can mitigate the shielding effect of residual cavitation bubbles resulting in a more efficient SWL treatment.

\section{Acknowledgments}

Research reported in this publication was supported by the National Institutes of Health under award number R01DK091267. The content is solely the responsibility of the authors and does not necessarily represent the official views of the National Institutes of Health.

\section{Author Disclosure Statement}

W.W.R., C.A.C., and T.L.H. have financial interests and consulting relationships with HistoSonics, Inc. A.P.D is an employee of HistoSonics, Inc. 


\section{References}

1. Duryea AP, Roberts WW, Cain CA, Tamaddoni HA, Hall TL. Acoustic bubble removal to enhance SWL efficacy at high shock rate: An in vitro study. J Endourol 2014;28:90-95.

2. Pishchalnikov YA, McAteer JA, Williams JC, Jr., Pishchalnikova IV, Vonderhaar RJ. Why stones break better at slow shockwave rates than at fast rates: In vitro study with a research electrohydraulic lithotripter. J Endourol 2006;20: 537-541.

3. Weir MJ, Tariq N, Honey RJ. Shockwave frequency affects fragmentation in a kidney stone model. J Endourol 2000;14: $547-550$.

4. Greenstein A, Matzkin H. Does the rate of extracorporeal shock wave delivery affect stone fragmentation? Urology 1999;54:430-432.

5. Paterson RF, Lifshitz DA, Lingeman JE, Evan AP, Connors BA, Fineberg NS, Williams JC, McAteer JA. Stone fragmentation during shock wave lithotripsy is improved by slowing the shock wave rate: Studies with a new animal model. J Urol 2002;168:2211-2215.

6. Pace KT, Ghiculete D, Harju M, Honey RJ, University of Toronto Lithotripsy Associates. Shock wave lithotripsy at 60 or 120 shocks per minute: A randomized, double-blind trial. J Urol 2005; 174:595-599.

7. Yilmaz E, Batislam E, Basar M, Tuglu D, Mert C, Basar H. Optimal frequency in extracorporeal shock wave lithotripsy: Prospective randomized study. Urology 2005;66:11601164.

8. Madbouly K, El-Tiraifi AM, Seida M, El-Faqih SR, Atassi $\mathrm{R}$, Talic RF. Slow versus fast shock wave lithotripsy rate for urolithiasis: A prospective randomized study. J Urol 2005; 173:127-130.

9. Delius M, Ueberle F, Eisenmenger W. Extracorporeal shock waves act by shock wave-gas bubble interaction. Ultrasound Med Biol 1998;24:1055-1059.

10. Philipp A, Delius M, Scheffczyk C, Vogel A, Lauterborn W. Interaction of lithotripter-generated shock waves with air bubbles. J Acoust Soc Am 1993;93:2496-2509.

11. Pishchalnikov YA, McAteer JA, Bailey MR, Pishchalnikova IV, Williams JC, Jr., Evan AP. Acoustic shielding by cavitation bubbles in shock wave lithotripsy (SWL). In: 17th International Symposium on Nonlinear Acoustics including the International Sonic Boom Forum 2006 18-22, July, 2005, State College, PA, AIP Conf. Proc., Vol. 838, No. 1, pp. 319-322. http://dx.doi.org/10.1063/1.2210369

12. Lokhandwalla M, Sturtevant B. Fracture mechanics model of stone comminution in ESWL and implications for tissue damage. Phys Med Biol 2000;45:1923.

13. Zhu S, Cocks FH, Preminger GM, Zhong P. The role of stress waves and cavitation in stone comminution in shock wave lithotripsy. Ultrasound Med Biol 2002;28: 661-671.

14. Zhong P, Chuong CJ, Preminger GM. Characterization of fracture toughness of renal calculi using a microindentation technique. J Mater Sci Lett 1993;12:1460-1462.

15. Duryea A, Roberts W, Cain C, Hall T. Controlled cavitation to augment SWL stone comminution: Mechanistic insights in vitro. IEEE Trans Ultrason Ferroelectr Freq Control 2013;60:301-309.
16. Flynn HG. Generation of transient cavities in liquids by microsecond pulses of ultrasound. J Acoust Soc Am 1982; 72:1926-1932.

17. Fowlkes JB, Crum LA. Cavitation threshold measurements for microsecond length pulses of ultrasound. J Acoust Soc Am 1988;83:2190-2201.

18. Pishchalnikov YA, McAteer JA, Pishchalnikova IV, Williams JC, Jr., Bailey MR, Sapozhnikov OA. Bubble proliferation in shock wave lithotripsy occurs during inertial collapse. In: 18th International Symposium on Nonlinear Acoustics, ISNA 187 10, July, 2008, Stockholm, Sweden, AIP Conf. Proc., Vol. 1022, No. 1, pp. 460-463. http://dx.doi.org/10.1063/1.2956259

19. Duryea A, Cain C, Roberts W, Tamaddoni H, Hall T. Active removal of residual bubble nuclei following a cavitation event. In: Ultrasonics Symposium (IUS), 2013 IEEE International 21-25, July, 2013, IEEE, Prague Convention Center (PCC), Prague, Czech Republic, pp. 1813-1816.

20. Duryea A, Cain C, Tamaddoni H, Roberts G, Hall T. Removal of residual nuclei following a cavitation event using low-amplitude ultrasound. IEEE Trans Ultrason Ferroelectr Freq Control 2014;61:1619-1626.

21. Simmons WN, Cocks FH, Zhong P, Preminger G. A composite kidney stone phantom with mechanical properties controllable over the range of human kidney stones. J Mech Behav Biomed Mater 2010;3:130-133.

22. Coleman AJ, Saunders JE, Choi MJ. An experimental shock wave generator for lithotripsy studies. Phys Med Biol 1989;34:1733.

23. Duryea AP, Roberts WW, Cain CA, Hall TL. Optically triggered solid state driver for shock wave therapy. In: 11th International Symposium on Therapeutic Ultrasound 11-13, April, 2011, New York, NY, USA, AIP Conf. Proc., Vol. 1481, No. 1, pp. 76-80. http://dx.doi.org/10.1063/1.4757314

24. Xi X, Zhong P. Improvement of stone fragmentation during shock-wave lithotripsy using a combined EH/PEAA shockwave generator-in vitro experiments. Ultrasound Med Biol 2000;26:457-467.

25. Zhou Y, Cocks FH, Preminger GM, Zhong P. Innovations in shock wave lithotripsy technology: Updates in experimental studies. J Urol 2004;172:1892-1898.

Address correspondence to: Hedieh Alavi Tamaddoni, MSE, BS Department of Biomedical Engineering University of Michigan 2131 Carl A. Gerstacker Building 2200 Bonisteel Boulevard Ann Arbor, MI 48109

E-mail: alavi@umich.edu

$\begin{aligned} & \text { Abbreviations Used } \\ \mathrm{ABC} & =\text { acoustic bubble coalescence } \\ \mathrm{EHL} & =\text { electrohydraulic lithotripter } \\ \mathrm{PRF} & =\text { pulse repetition rate } \\ \mathrm{SW} & =\text { shockwave } \\ \mathrm{SWL} & =\text { shockwave lithotripsy }\end{aligned}$

